\title{
A CLINICAL STUDY ON MANAGEMENT OF POST-OPERATIVE PAIN AND QUALITY OF LIFE OF OSTEOARTHRITIS PATIENTS AFTER TOTAL KNEE ARTHROPLASTY
}

\author{
TAMILSELVAN T*, KALAIVANI H
}

Department of Pharmacy Practice, Swamy Vivekanandha College of Pharmacy, Tiruchengode, Tamil Nadu, India. Email: tamilrx@gmail.com

Received: 21 November 2017, Revised and Accepted: 19 December 2017

\begin{abstract}
Objective: The objective of this study is to assess the effect of pregabalin in the management of post-operative pain and the quality of life (QOL) of osteoarthritis (OA) patients after total knee arthroplasty.

Methods: This prospective observational study was conducted in the Department of Orthopedics. A total of 96 patients were divided into two groups. Group A consists of 50 patients with the treatment of nonsteroidal anti-inflammatory drugs (NSAIDs) and Group B consists of 46 patients with the treatment of pregabalin with NSAIDs. The study subjects were followed once in 60 days for 6 months, and they were asked to answer the visual analog scale (VAS) and knee injury and OA outcome score (KOOS) questionnaire. The effect of the treatment was assessed by comparing the baseline score with follow-up score.
\end{abstract}

Results: Our study result showed that the pain score of Group B in VAS at $2^{\text {nd }}$ follow-up was $2.56 \pm 0.34$ and K00S pain score was $92.73 \pm 3.45$ (p<0.01). The QOL score was improved significantly to $81.56 \pm 5.29(\mathrm{p}<0.01)$ as compared to Group A.

Conclusion: The study concluded that pregabalin with NSAIDs group patients showed a better improvement in pain, symptoms, and QOL within short duration as compared to NSAIDs alone used group.

Keywords: Osteoarthritis, Total knee arthroplasty, Pregabalin, Nonsteroidal anti-inflammatory drugs, Visual analog scale.

(C) 2018 The Authors. Published by Innovare Academic Sciences Pvt Ltd. This is an open access article under the CC BY license (http://creativecommons. org/licenses/by/4. 0/) DOI: http://dx.doi.org/10.22159/ajpcr.2018.v11i4.23768

\section{INTRODUCTION}

Osteoarthritis (OA) is a common, progressive disorder affecting primarily weight-bearing diarthrodial joints [1]. Worldwide, OA is estimated to be the fourth leading cause of disability. Recent reports, over $40 \%$ of the Indian population in the age group of 70 or above suffer from OA. Good pain control after surgery is important to prevent negative outcomes such as tachycardia, hypertension, and myocardial ischemia and decrease in alveolar ventilation, poor wound healing, and Insomnia [2].

Total knee arthroplasty (TKA) is an effective treatment for end-stage knee $\mathrm{OA}$, but the optimal management of post-operative pain remains controversial. TKA is often cited as one of the most painful known procedures. Approximately $20 \%$ of the patients with OA of the knee do not want to undergo TKA because of the expectancy of high levels of pain. After TKA, the pain may also inhibit early rehabilitation to mobilize the knee joint [3].

Nonsteroidal anti-inflammatory drugs (NSAIDs) are often considered to be the preferred first-line treatment for OA. The use of non-opioid drugs during the pre-operative period can reduce excess intraoperative opioid use. The use of pre-operative NSAIDs and cyclooxigenase-2 (COX-2) inhibitors also has a significant effect on opioid requirements following surgery. This has been referred to as an "opioid-sparing effect." The clinical significance of this may be the reduction of opioid-related side effects, improved analgesia, and better patient satisfaction [4].

In addition to NSAIDs and COX-2 inhibitors, anti-neuropathic drugs are playing a role in the treatment of post-operative pain. Drugs such as gabapentin and pregabalin, intended for seizures and neuropathic pain syndromes, can inhibit central neuronal sensitization. Pregabalin was also found to have a synergistic effect with COX-2 inhibitors in clinical studies involving patient undergoing spinal fusion. The combination of the two drugs reduced post-operative pain, morphine consumption at $24 \mathrm{~h}$, and opioid-induced side effects. Improvements in outcome were greater when these drugs were used in combination than either of the drugs used alone [5].

\section{METHODS}

A prospective observational study was carried out in the Department of Orthopedics in a Tertiary Care Hospital. The study was approved by the Institutional Ethical Committee. The duration of study was 1 year from November 2016 to October 2017. The patient's history was collected from patient case records, designed data entry form and pain questionnaire, medication charts, laboratory reports, and patient/ patient caretaker's interview. The effect of the treatment was assessed using visual analog scale (VAS) [6] and knee injury and OA outcome score (KOOS) questionnaire [7].

Patients were eligible for the study if patients with 40-75 years of age, both male and female patients, patients with the treatment of NSAIDs or pregabalin with NSAIDs, and patients who underwent TKA. Patients were excluded if patients have an allergy or intolerance to any of the study drugs, severe liver failure, and heart or renal failure patients, patients with a history of opioid medication usage, and patients with the bleeding disorder.

Randomly selected patients were assigned to receive study medication. A total of 96 patients are divided into two groups. Group A consists of 50 patients with the treatment of NSAIDs paracetamol $325 \mathrm{mg}+$ aceclofenac $100 \mathrm{mg}$ and Group B consists of 46 patients with the treatment of pregabalin $75 \mathrm{mg}+$ NSAIDs (paracetamol 325 mg+aceclofenac $100 \mathrm{mg}$ ). The patient demographics data were collected using the data entry form. The baseline score of pain, 
Table 1: Patient demographics

\begin{tabular}{ll}
\hline Patient demographics & Number of patient (\%) \\
\hline Age (years) & $9(9.40)$ \\
$40-49$ & $23(24.00)$ \\
$50-59$ & $46(48.00)$ \\
$60-69$ & $18(18.75)$ \\
$70-79$ & $40(41.66)$ \\
Gender & $56(58.34)$ \\
Male & \\
Female & $63(65.62)$ \\
BMI & $33(34.38)$ \\
Normal weight & \\
Overweight &
\end{tabular}

Table 2: Comparison of VAS score

\begin{tabular}{llll}
\hline VAS score & Baseline & Follow-up-1 & Follow-up-2 \\
\hline Group A & $5.7 \pm 0.58$ & $5.18 \pm 0.59$ & $4.42 \pm 0.86^{* *}$ \\
Group B & $5.6 \pm 0.93$ & $3.97 \pm 0.46$ & $2.56 \pm 0.34^{* *}$ \\
\hline
\end{tabular}

VAS-Visual analog scale, ${ }^{* *} \mathrm{p}<0.01$

symptoms, activity of daily living, sport and recreation function, and quality of life (QOL) were assessed using KOOS questionnaire and VAS. The study subjects were followed once in 60 days for 6 months, and they were asked to answer the same questionnaire. The initial score was considered as a baseline. The study subjects were followed once in 60 days and they were asked to answer the same questionnaire on every follow-up. The effect of the treatment was assessed by comparing the baseline score with follow-up score. The QOL of the patient before and after the treatment was assessed by KOOS

\section{Statistical analysis}

All results were analyzed using the computerized statistical package of Graph Pad InStat Version 3.10. Values were represented as mean standard deviation. Scores of KOOS questionnaire and VAS were compared using the Student's t-test. $\mathrm{p}<0.01$ was considered as statistically significant at $95 \%$ confidence interval.

\section{RESULTS}

In this study, 96 patients were randomly selected and divided into two Groups A and B. Among 96 patients, $9.40 \%$ (9) were in the age group of $40-49$ years, $24 \%$ (23) were in the age group of $50-59$ years, $48 \%$ (46) were in the age group of $60-69$ years, and $18.75 \%$ (18) were in the age group of 70-79 years. The mean age of the study population was 61.66-7.56 years (range 40-79). Males were $41.66 \%$ (40) and females were $58.34 \%$ (56). As per the BMI result, $65.62 \%$ (63) were in normal body weight and $34.38 \%$ (33) were in over body weight (Table 1).

\section{COMPARISON OF EFFECT OF GROUP A AND GROUP B FOR OA AFTER TKA ON VAS}

On VAS pain score, there was a significant difference between baseline $5.7 \pm 0.58$ and $2^{\text {nd }}$ follow-up $4.42 \pm 0.86$ in Group A. In Group B, baseline score was $5.6 \pm 0.93$ and $2^{\text {nd }}$ follow-up $2.56 \pm 0.34$. ( $\left.\mathrm{p}<0.01\right)$. Group B mean score difference was high as compared to Group A (Table 2).

\section{ASSESSMENT OF GROUP A USING KOOS QUESTIONNAIRE}

KOOS mean pain score at baseline was $62.78 \pm 7.92$ and at $2^{\text {nd }}$ follow-up was $79.06 \pm 6.68$. The mean score of symptoms at follow-up- 2 was significantly improved as compared with other parameters $(78.56 \pm 9.80)$. The mean difference was statistically significant from baseline $(\mathrm{p}<0.01)$ (Table 3).

\section{ASSESSMENT OF GROUP B USING KOOS QUESTIONNAIRE}

KOOS mean pain score at baseline was $67.19 \pm 5.73$ and $2^{\text {nd }}$ follow-up $92.73 \pm 3.45$. The mean score of QOL at follow-up-2 was significantly
Table 3: Assessment of Group A using KOOS questionnaire

\begin{tabular}{llll}
\hline Parameters & Baseline & Follow-up-1 & Follow-up-2 \\
\hline Pain & $62.78 \pm 7.92$ & $70.84 \pm 6.70$ & $79.06 \pm 6.68^{* *}$ \\
Symptoms & $61.28 \pm 11.72$ & $68.36 \pm 11.84$ & $78.56 \pm 9.80^{* *}$ \\
ADL & $76.42 \pm 6.46$ & $82.42 \pm 5.41$ & $88.14 \pm 3.55^{* *}$ \\
SRF & $91.3 \pm 2.82$ & $92.7 \pm 3.61$ & $93.8 \pm 3.28^{* *}$ \\
QOL & $57.7 \pm 9.63$ & $59.38 \pm 8.80$ & $64.92 \pm 6.00^{* *}$ \\
\hline
\end{tabular}

KOOS: Knee injury and osteoarthritis outcome score, SRF: Sport and recreation function, QOL: Quality of life, ${ }^{* *} \mathrm{p}<0.01$. ADL: Activities of daily living

Table 4: Assessment of Group B using KoOS questionnaire

\begin{tabular}{llll}
\hline Parameters & Baseline & Follow-up-1 & Follow-up-2 \\
\hline Pain & $67.19 \pm 5.73$ & $84.96 \pm 4.02$ & $92.73 \pm 3.45^{* *}$ \\
Symptoms & $67.87 \pm 8.35$ & $83.56 \pm 6.23$ & $89.23 \pm 3.59^{* *}$ \\
Activity of daily living & $80.21 \pm 5.40$ & $89.52 \pm 2.14$ & $94.97 \pm 2.71^{* *}$ \\
SRF & $92.39 \pm 5.55$ & $93.58 \pm 4.03$ & $92.60 \pm 2.52$ \\
QOL & $57.5 \pm 8.18$ & $71.60 \pm 3.49$ & $81.56 \pm 5.29^{* *}$ \\
\hline
\end{tabular}

KOOS: Knee injury and osteoarthritis outcome score, SRF: Sport and recreation function, QOL: Quality of life, ${ }^{* *} \mathrm{p}<0.01$

improved as compared with other parameters $81.56 \pm 5.29$. The mean difference was statistically significant from baseline $(\mathrm{p}<0.01)$ (Table 4).

\section{COMPARISON OF EFFECT OF TREATMENT}

The Group B treatment was more effective in reduction of pain and symptoms as compared with Group A. The mean difference of pain and symptoms score between Group A and Group B was statistically significant $(\mathrm{p}<0.01)$. The improvement of QOL was high in Group B as compared to Group A (Table 5).

\section{DISCUSSION}

Among 96 patients, of which 46 patients were in the age group of 60-69 years. The mean age of this study population was $61.65 \pm 7.55$ years. A study conducted by Ohtori et al. showed that the mean age of patients was $70.0 \pm 8$ years [8,9]. As per the study results, female patients $(58.34 \%)$ were more suffered by OA than male $(41.66 \%)$ patients. The majority of patients were in our study was normal weights. Earlier study was reported that the majority of patients were having over body weight [10].

As per assessment, by VAS, Group B was found to have a significant reduction in pain intensity than Group A patients. Mathiesen et al. have reported that pregabalin administration could reduce the VAS score at $24 \mathrm{~h}$ compared with placebo postoperatively [11].

In this study, oral administration of pregabalin with NSAIDs was more effective in reducing pain as compared with NSAIDs alone in post-operative cases. Fassoulaki et al. said that the administration of gabapentin to the woman undergoing total abdominal hysterectomy did not reduce acute post-operative pain. However, there was a decrease in pain at 1 month postoperatively [12].

Group B was also effective in impaired active and passive range of motion (ROM) after TKA as compared with Group A patients. The mean difference of KOOS symptoms score was statistically significant $(\mathrm{p}<0.01)$ and result indicated that the symptom was decreased and knee flexion was improved as compared with Group A. Chiu et al. resulted that oral pre-operative administration of pregabalin improved knee flexion and ROM after TKA $[13,14]$.

The mean difference of activities of daily living (ADL) and QOL After TKA between Group A and B was statistically significant at final followup. The result indicates that the ADL and QOL were improved in Group B patients as compared with Group A patients. Based on the study findings, Group B patients had reduced pain and improved QOL of TKA as compared with Group A patients. 
Table 5: Comparison of effect of treatment

\begin{tabular}{lll}
\hline Parameters & Group A & Group B \\
\hline Pain & $79.06 \pm 6.68$ & $92.73 \pm 3.45^{* *}$ \\
Symptoms & $78.56 \pm 9.80$ & $89.23 \pm 3.59^{* *}$ \\
ADL & $88.14 \pm 3.55$ & $94.97 \pm 2.71^{* *}$ \\
SRF & $93.8 \pm 3.28$ & $92.60 \pm 2.52$ \\
QOL & $64.92 \pm 6.00$ & $81.56 \pm 5.29^{* *}$ \\
\hline
\end{tabular}

KOOS: Knee injury and osteoarthritis outcome score, SRF: Sport and recreation function, QOL: Quality of life, ${ }^{* *} \mathrm{p}<0.01$. ADL: Activities of daily living

\section{CONCLUSION}

The present study showed that pregabalin with NSAIDs group was more effective in reducing pain after TKA as compared with NSAIDs group. As per the study, pregabalin with NSAID group results revealed that a better improvement in OA symptoms, ADL and QOL within a short duration. Regular follow-up and good patient compliance may improve the QOL of patients in the day-to-day activities. Long-term treatment of pregabalin may reduce the dependence of analgesics and decrease OArelated neuropathic pain incidence.

\section{ACKNOWLEDGMENT}

We would like to acknowledge Dr. S. Arthanareeswaran (Executive Director) and Dr. S. Kumaravel (Orthopedic Surgeon), Vivekanandha Medical Care Hospital, Tiruchengode, Tamil Nadu, India, for giving us an opportunity to conduct the study and guiding our research work.

\section{CONFLICT OF INTEREST}

The author has declared no conflict of interest.

\section{REFERENCES}

1. Wells BG, Dipiro JT, Schwinghammer TL, Dipiro CV. Osteoarthritis In: Pharmacotherapy Handbook. $9^{\text {th }}$ ed. New York: McGraw-Hill Professional Publishing; 2014. p. 9-10.
2. Vadivelu N, Mitra S, Narayan D. Recent advances in postoperative pain management. Yale J Biol Med 2010;83:11-25.

3. Sigirci A. A new method for pain management in total knee arthroplasty: Intraoperative local anesthetic application in addition to one shot femoral block. Int J Clin Exp Physiol 2016;3:17-21.

4. Horlocker TT. Pain management in total knee Arthroplasty: A historical review. J Am Acad Orthop Surg 2006;14:126-35.

5. Gandhi K. Multimodal pain management techniques in hip and knee arthroplasty. J N Y Sch Reg Anesth 2009;13:1-10.

6. Selvan T, Kingston RM, Sundara MN, Mathew EM. A clinical study on glucosamine sulfate versus combination of glucosamine sulfate and NSAIDs in Mild to moderate knee osteoarthritis. Sci World J 2012;2012:1-5.

7. Peer MA, Lane J. The knee injury and osteoarthritis outcome score (KOOS): A Review of its psychometric properties in people undergoing total knee arthroplasty. J Orthop Sports Phys Ther 2013;3:20-8.

8. Ohtori S, Inoue G, Orita S, Takaso M, Eguchi Y, Ochiai N, et al. Efficacy of combination of meloxicam and pregabalin for pain in knee osteoarthritis. Yonsei Med J 2013;54:1253-8.

9. Dwicandra NM, Setiadi AP. Comparison of pain score in osteoarthritis patients treated with a combination of diacerein and meloxicam and meloxicam alone. Int J Pharm Pharm Sci 2017;9:69-73.

10. Buvanendran A, Kroin JS, Della Valle CJ, Kari M, Moric M, Tuman KJ, et al. Perioperative oral pregabalin reduces chronic pain after total knee arthroplasty: A prospective, randomized, controlled trial. Anesth Analg 2010;110:199-207.

11. Mathiesen O, Jacobsen LS, Holm HE, Randall S. Pregabalin and dexamethasone for postoperative pain control: A randomized controlled study in hip arthroplasty. Br J Anesth 2008;101:535-41.

12. Fassoulaki A, Stamatakis E, Petropoulos G, Siafaka I, Hassiakos D, Sarantopoulos C. Gabapentin attenuates late but not acute pain abdominal pain after abdominal hysterectomy. Eur J Anaesthesiol 2006;23:136-41.

13. Jena SS, Jena M, Patro N, Mishra S, Panda M, Dash M. Patterns of prescription and ADR monitoring of drugs in the management of neuropathic pain in a tertiary care teaching hospital. Int J Pharm Pharm Sci 2014;6:246-51.

14. Chiu KY, Ng TP, Tang WM, Yau WP. Review article: Knee flexion after total knee arthroplasty. J Orthop Surg 2002;10:194-243. 